\title{
Nutritional Interventions Using Functional Foods and Nutraceuticals to Improve Inflammatory Bowel Disease
}

\author{
Dong Wook Shin ${ }^{1}$ and Beong Ou Lim ${ }^{1,2}$ \\ ${ }^{1}$ College of Biomedical and Health Science, Konkuk University, Chungju, Korea. \\ ${ }^{2}$ Research Institute of Inflammatory Disease, Konkuk University, Chungju, Korea.
}

\begin{abstract}
The gastrointestinal tract, the second largest organ in the body, plays an important role in nutrient and mineral intake through the intestinal barrier. Dysfunction of intestinal permeability and related disorders commonly occur in patients with inflammatory bowel disease (IBD), one of the health problems in the Western societies that are considered to be mainly due to the Western diet. Although the exact etiology of IBD has not been elucidated, environmental and genetic factors may be involved in its pathogenesis. Many synthetic or biological drugs, such as 5-aminosalicylic acid corticosteroids as antiinflammatory drugs, have been used clinically to treat IBD. However, their long-term use exhibits some adverse health consequences. Therefore, many researchers have devised alternative therapies to overcome this problem. Many studies have revealed that some functional nutrients in nature can relieve gastrointestinal inflammation by controlling proinflammatory cytokines. In this study, we review the ability of functional nutraceuticals such as phytochemicals, fatty acids, and bioactive peptides in improving IBD by regulating its underlying pathogenic mechanisms.
\end{abstract}

\section{KEYWORDS: • functional foods $\bullet$ nutraceuticals $\bullet$ inflammatory bowel disease}

\section{INTRODUCTION}

I NFLAMMATORY BOWEL DISEASE (IBD), a chronic gastrointestinal disease characterized by recurrent inflammation in the intestine and severe mucosal damage, is generally divided into two categories: ulcerative colitis (UC) and Crohn's disease (CD). These symptoms include diarrhea, nausea, abdominal pain, rectal bleeding, and weight loss. ${ }^{1}$ Recent research has shown a correlation between nutrition and risk of developing IBD in countries with a fat-andprotein-rich diet similar to that observed in Western countries. ${ }^{2}$ We speculate that genetic susceptibility, percentage of intestinal microorganisms, and types of nutrition play important roles in the pathogenesis of IBD. ${ }^{3}$

Nutritional interventions aimed at potentially reducing nutritional risk are attracting attention as a treatment method for IBD that induces prolonged disease relief. ${ }^{4}$ Recent studies suggest that fruits, vegetables, probiotics, and fiber can significantly decrease the symptoms of IBD through anti-inflammatory effects. ${ }^{5-9}$ In contrast, because high-fat foods and high-carbohydrate foods are known to contribute to the development of IBD, limiting the intake of these foods helps improve IBD. ${ }^{10,11}$ Functional foods and bioactive natural compounds have been key research topics to provide

Manuscript received 17 February 2020. Revision accepted 4 August 2020.

Address correspondence to: Beong Ou Lim, PhD, Research Institute of Inflammatory Disease, Konkuk University, Chungju 380-701, Korea, E-mail: beongou@kku.ac.kr potential treatments for improving IBD. Several groups have suggested that active materials derived from various plants exert potent anti-inflammatory effects against IBD through various mechanisms. ${ }^{12-15}$ These types of research will continuously provide much evidence and scientific rationale about the use of a healthy diet or functional foods to improve or ameliorate the symptoms of IBD. Therefore, in this study we review the beneficial effects of various dietary nutrients as a treatment method for improving IBD.

\section{CHARACTERISTICS OF UC AND CD}

Both UC and CD share a common symptom but have different histopathological and symptomatic characteristics. UC is characterized by mucosal or submucosal inflammation, usually in the rectum and sometimes in the colon, with symptoms of abdominal pain, diarrhea, and bleeding. CD can occur in the gastrointestinal tract and cause intestinal granuloma, fistula, and stenosis, but is caused by inflammation of the intestinal tract and colon that leads to $a b-$ dominal pain, diarrhea, fever, appetite, weight loss, and intermittent anal fissures. Although the pathogenesis of IBD has not been clarified to date, various dietary, immunological, environmental, genetic, and microbial factors have been identified as causes. ${ }^{14}$ Complex interactions of genetic, environmental, microbiological, and immunological factors can simultaneously induce various cytokines, activating the mucosal immune responses. ${ }^{15}$ Cytokines, which are mainly produced by immune cells, transmit specific signals that 
interact with cells, causing local and systemic inflammatory reactions. Unlike healthy individuals, patients with IBD develop imbalances between intestinal homeostasis and inflammatory cytokines that induce an unusual increase in the proinflammatory cytokine activity in the mucosa, dysfunction of the epithelial barrier, and damage to one's intestinal permeability. ${ }^{16}$

\section{POSSIBLE MOLECULAR TARGETS IN IBD}

\section{Tumor necrosis factor- $\alpha$}

A proinflammatory cytokine, tumor necrosis factor alpha (TNF- $\alpha$ ), which is primarily secreted by activated macrophages, is elevated in patients with IBD. ${ }^{17}$ The increased production of TNF- $\alpha$ triggers mucosal inflammation, and causes the impairment of the intestinal barrier by malfunctional tight junctions as well as cell death of intestinal epithelial cells (IECs) ${ }^{18}$ The excess level of TNF- $\alpha$ initiates a positive feedback loop by causing the secretion of interleukin (IL)- $1 \beta$ and IL-6, which are chiefly produced by leukocytes, mast cells, macrophages, and neutrophils. ${ }^{19,20}$ IL-6 activates Th17 and Th2 responses, which aggravate the inflammation. This cytokine cascade leads to harmful effects on the barrier function.

\section{Nuclear factor-kappa B}

Nuclear factor-kappa B (NF- $\kappa \mathrm{B})$ plays a key role in mediating inflammation by regulating the expression of many proinflammatory genes. ${ }^{21} \mathrm{NF}-\kappa \mathrm{B}$ signaling is dysregulated in IBD, which causes excessive inflammation in IBD patients. Among canonical or noncanonical, the activation of the canonical NF- $\kappa$ B pathway is observed in IBD and related to the acute expression of cyclooxygenase 2 $(\mathrm{COX}-2), \mathrm{IL}-1 \beta$, and IL-6. ${ }^{22,23}$

\section{Interleukin-6}

IL-6 is found to increase in the inflammatory tissues and serum of patients with IBD. A humanized monoclonal antibody (tocilizumab) against IL-6, approved for the treatment of rheumatic conditions, was reportedly successful in case reports of IBD. ${ }^{24}$ However, according to some results of siplizumab treatment in patients with IBD, the rate of intestinal perforation appeared to be relatively increased..$^{25}$ To date, other antibodies against IL-6 have been developed and studied in preclinical studies but have not yet been evaluated in clinical trials.

\section{Interleukin-22}

IL-22 is reportedly more highly expressed in patients with CD. ${ }^{26}$ IL-22 has a protective role, but induces inflammation under certain conditions. ${ }^{27} \mathrm{~A}$ previous study reported that IL-22-mediated gene therapy can inhibit inflammatory responses and mitigate tissue damage. ${ }^{28}$ In addition, the clinical relevance of IL-22 for IBD has been well described. Mucosal healing, the current therapeutic target for IBD, can be enhanced by IL-22. IL-22 increases the expression level of MUC1, a core protein of the mucus layer. ${ }^{29,30}$ Patients with IBD lacking IL-1 $\beta 0$ R2, an IL-22 receptor, do not express the glycosylated protein MUC1 ${ }^{31}$ Therefore, since IL-22 is linked to the main function of the intestinal barrier, future studies are needed to confirm its role in IBD and its therapeutic potential.

\section{CURRENT CLINICAL TREATMENTS FOR CURING PATIENTS WITH IBD}

Since we do not know the exact underlying pathogenic mechanism of IBD, there is currently no effective treatment for IBD. The current goal of IBD treatment is to significantly reduce the inflammation that causes symptoms and signs to achieve symptomatic relief as well as long-term remission and a decreased risk of complications.

\section{Anti-inflammatory drugs}

Anti-inflammatory drugs are the first to be prescribed clinically for the treatment of IBD. These drugs are usually corticosteroids and aminosalicylates such as balsalazide (Colazal), mesalamine (Asacol HD, Delzicol, others), and olsalazine (Dipentum). ${ }^{32}$ These drugs improve the symptoms of IBD by blocking or reducing proinflammatory cytokines. These drugs are differently prescribed depending on the affected colon site.

\section{Immune system suppressors}

These drugs work by suppressing the immune response to released inflammatory substances in the intestines. Interestingly, a combination of these drugs works better than a single drug for some people. These immunosuppressant drugs are cyclosporine (Gengraf, Neoral, Sandimmune), azathioprine (Azasan, Imuran), mercaptopurine (Purinethol, Purixan), and methotrexate (Trexall). ${ }^{33}$

\section{Biologics}

The use of biological drugs derived from living biological resources have become widespread and treat IBD by neutralizing a protein produced by the immune system. The representative biologics for IBD are TNF- $\alpha$ inhibitors such as infliximab (Remicade), adalimumab (Humira), and golimumab (Simponi). Other types of therapeutic biologics such as natalizumab (Tysabri) against the cell adhesion molecule $\alpha 4$-integrin, vedolizumab (Entyvio) against integrins, and ustekinumab (Stelara), an IL-12 and IL-23 antagonist, are approved for treating IBD. ${ }^{34}$

\section{Antibiotics}

In addition to other medications or cases of infection, antibiotics such as ciprofloxacin (Cipro) and metronidazole (Flagyl) are generally prescribed in CD occurring around the anus. ${ }^{35}$ 


\section{FUNCTIONAL NUTRACEUTICALS FOR IMPROVING IBD}

Despite providing some temporary symptomatic relief, the current drugs may cause serious side effects. ${ }^{36}$ Biologics, which have recently become the focus of treatment for IBD, are expensive compared with other drugs. Therefore, we will review alternative IBD therapies by utilizing natural products, which are relatively safe and inexpensive.

Nutraceuticals means foods or food supplements manufactured or processed to potentiate functional bioactive ingredients with pharmacological properties. Molecular, biological, and cellular mechanisms of natural bioactive compounds for nutraceuticals have been continuously elucidated. Some researchers have reported that various substances, the main components of functional foods capable of improving IBD, can alleviate IBD symptoms by suppressing the inflammatory pathways ${ }^{5-7}$ (Table 1 ).

\section{Phytochemicals}

Phytochemicals are generally derived from the primary and secondary metabolisms in plants as part of their survival mechanisms against pathogens. These chemicals possess important physiological functions against oxidative stress and the inflammatory response. Phytochemicals can be classified into major categories, such as alkaloids, and polyphenols, which include flavonoids and phenolic acids.

Anthocyanin-rich bilberry extract decreases the expression levels of IFN- $\gamma$ and TNF- $\alpha$ but increases those of the immunoregulatory cytokine IL-10 and Th17 cell-specific cytokine IL-22. ${ }^{37}$ Kaempferol decreased IL-6, IL-1 $\beta$, TNF- $\alpha$, inducible nitric oxide synthase (iNOS), COX-2 levels, nitric oxide (NO), prostaglandin E2 (PGE2), and colonic mucosa myeloperoxidase (MPO) activity in colitis mice model treated with dextran sodium sulfate (DSS).$^{38}$ Quercetin reduced MPO activity, increased glutathione (GSH) content, and reduced colon impairment in a colitis rat model treated with acetic acid. ${ }^{39}$ Isoquercitrin downregulated COX-2 and iNOS expressions in a DSS-induced acute colitis model in rats. ${ }^{40}$ Apigenin significantly decreased iNOS, COX-2, IL- $1 \beta$, and TNF- $\alpha$ production in DSS-induced colitis in mice. ${ }^{41}$

Hesperidin showed reduced MPO activity and IL-6 levels. ${ }^{42}$ Poncirin and its metabolite ponciretin improved the symptom of colitis in mice treated with $2,4,6-$ trinitrobenzene sulfonic acid (TNBS) by reducing NF- $\kappa$ B activation. ${ }^{43}$ Myricetin contributes to weight loss, improving histological scores, and the suppression of $\mathrm{NO}$, MPO, IL- $1 \beta$, and IL- 6 but enhances activities of superoxide dismutase and glutathione peroxidase. ${ }^{44}$ Naringenin increased the content of colonic mucus and reduced interferon (INF)- $\gamma$, IL-1 $\beta$, IL-6, PGE2, NO, and macrophage inflammatory protein in a colitis mouse model. ${ }^{45,46}$ Naringin ameliorated colonic mucosal damage and ulcer lesions by increasing superoxide dismutase and GSH levels. ${ }^{47}$

Apple polyphenols significantly reduced the mRNA expression of IL- $1 \beta$ and TNF- $\alpha$ in a colitis mouse model. ${ }^{48}$ Resveratrol, a representative polyphenol found in red grapes and several berry species, has modulatory effects on the oxidative process and inflammatory pathways as well as antiaging effects. ${ }^{49} \mathrm{~A}$ previous study reported that resveratrol decreased NF- $\kappa$ B activation, and downregulated activation of STAT3 in DSS-induced mouse. ${ }^{50}$ A recent study demonstrated that resveratrol ameliorated inflammation and significantly reduced expression levels of TNF- $\alpha$ and NF- $\kappa$ B in patients with UC compared with the control group. ${ }^{51}$

Curcumin, the principal curcuminoid derived from turmeric, has been used medically in some Eastern Asian countries. A previous study demonstrated that curcumin improved the tissue status of the colon by inhibiting STAT3 activity and decreasing TNF- $\alpha$ and IL- $1 \beta$ expressions. ${ }^{52}$ Curcumin also significantly inhibited the activation of NOD-like receptor pyrin domain-containing 3 (NLRP3) inflammasome by DSS treatment and ameliorate DSSinduced colitis in mice. ${ }^{53}$

Sophocarpine and sophoridine, isolated from Sophora alopecuroides, have been used as traditional Chinese medicine for treating rheumatism. ${ }^{54,55}$ Studies on DSS-induced murine colitis showed that sophocarpine $(15,30$, or $60 \mathrm{mg} / \mathrm{kg}$ body weight) decreased levels of IL- $1 \beta$, IL- 6 , and colonic MPO activity, and improved the symptom of DSSinduced colitis. ${ }^{56}$

Berberine, a well-known representative isoquinoline alkaloid that is isolated from Coptis chinensis and Phellodendron chinense, reportedly can protect against colitis by controlling the gut microbiota, the innate and adaptive immune responses, and the function of the intestinal barrier. ${ }^{57}$ Another study demonstrated that berberine significantly decreased levels of IFN- $\gamma$, IL-17, IL-6, IL- $1 \beta$, and TNF- $\alpha$ in the local colon but increased IL-22 levels. ${ }^{58}$

Isatin (6 and $25 \mathrm{mg} / \mathrm{kg}$ of body weight), obtained from Isatis tinctoria, protects against TNBS-induced gut mucosa injury in rats by reducing the expression level of COX-2, PGE2, and TNF- $\alpha$ and enhancing IL-10 levels. ${ }^{59}$ Tryptanthrin, which is obtained from Polygonum tinctorium, can treat inflammation, allergy, and cancer. ${ }^{60}$ Several studies have revealed that tryptanthrin treatment significantly attenuated colonic mucosal injury by decreasing the production of PGE2, TNF- $\alpha$, and NO in the TNF- $\alpha / \mathrm{NF}-\kappa$ B and IL-6/STAT3 signaling pathways in a colitis model treated with DSS. ${ }^{61,62}$

A previous study has demonstrated that caffeine, a natural xanthine alkaloid, relieved colitis symptoms by decreasing the expression level of chitinase 3-like 1 in the IECs. ${ }^{63}$

Ginsenoside $\operatorname{Rg} 1$ and ginsenoside Rh1 could inhibit colon shortening, MPO activity, the expression levels of IL- $1 \beta$, IL-17, TNF- $\alpha$, and restores the imbalance of Th17/Treg. ${ }^{64}$ Ginsenoside Rd alleviates colon inflammatory symptoms by inhibiting the NLRP3 inflammasome in a dose-dependent manner. ${ }^{65}$ Ginsenoside Re suppressed NF- $\kappa \mathrm{B}$ activity and antagonized the decrease of intestinal barrier function in IBD. ${ }^{66}$ Astragaloside IV, a cycloartane triterpene saponin, stimulated nuclear translocation of $\beta$-catenin and induced mucosal healing in colitis by regulating energy metabolism. ${ }^{67}$ Saponins improve intestinal health by inhibiting excessive inflammation by interaction with inflammatory pathways such as the NF- $\kappa \mathrm{B}$, MAPK, and TLR4 pathways. 


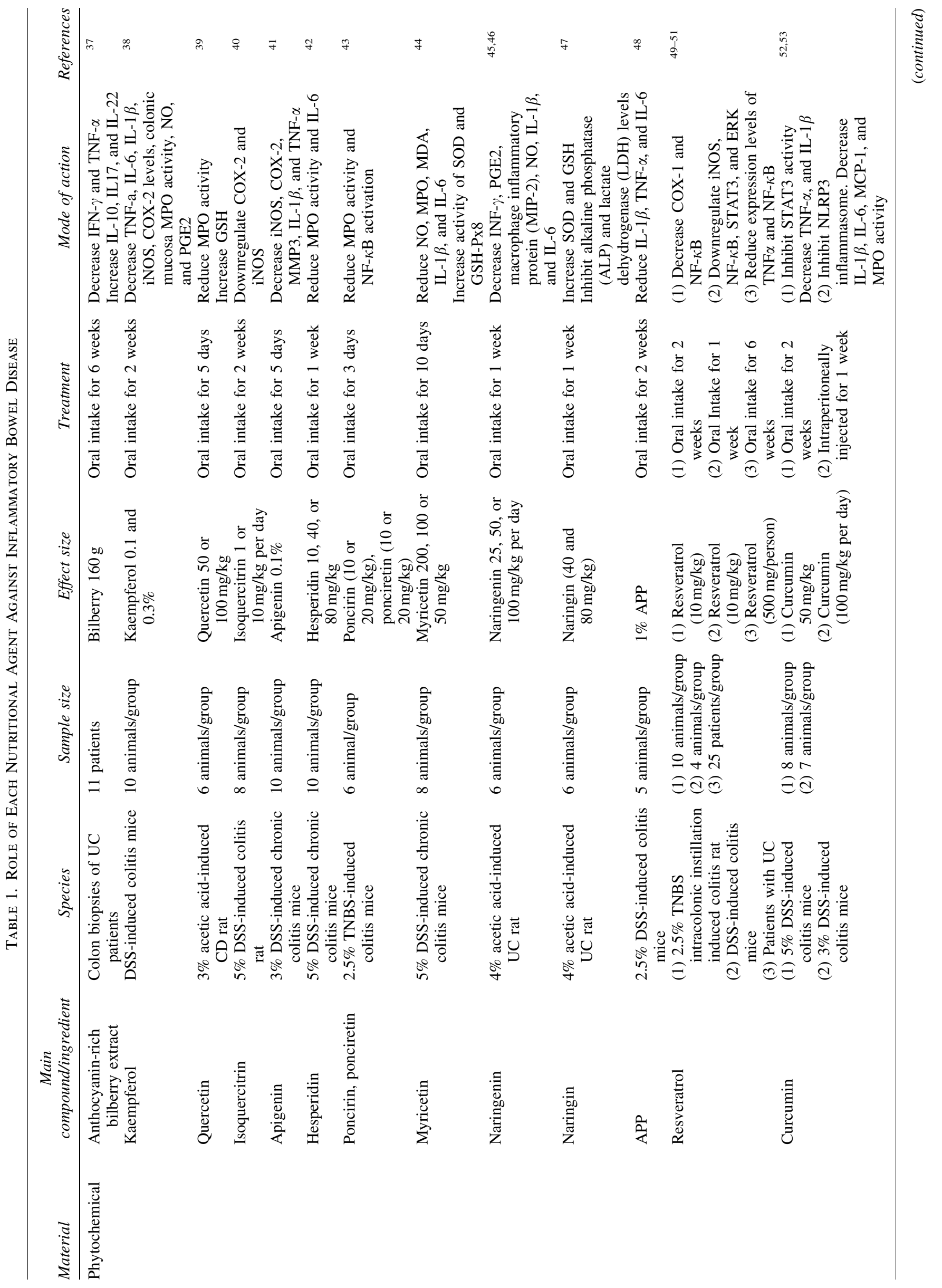




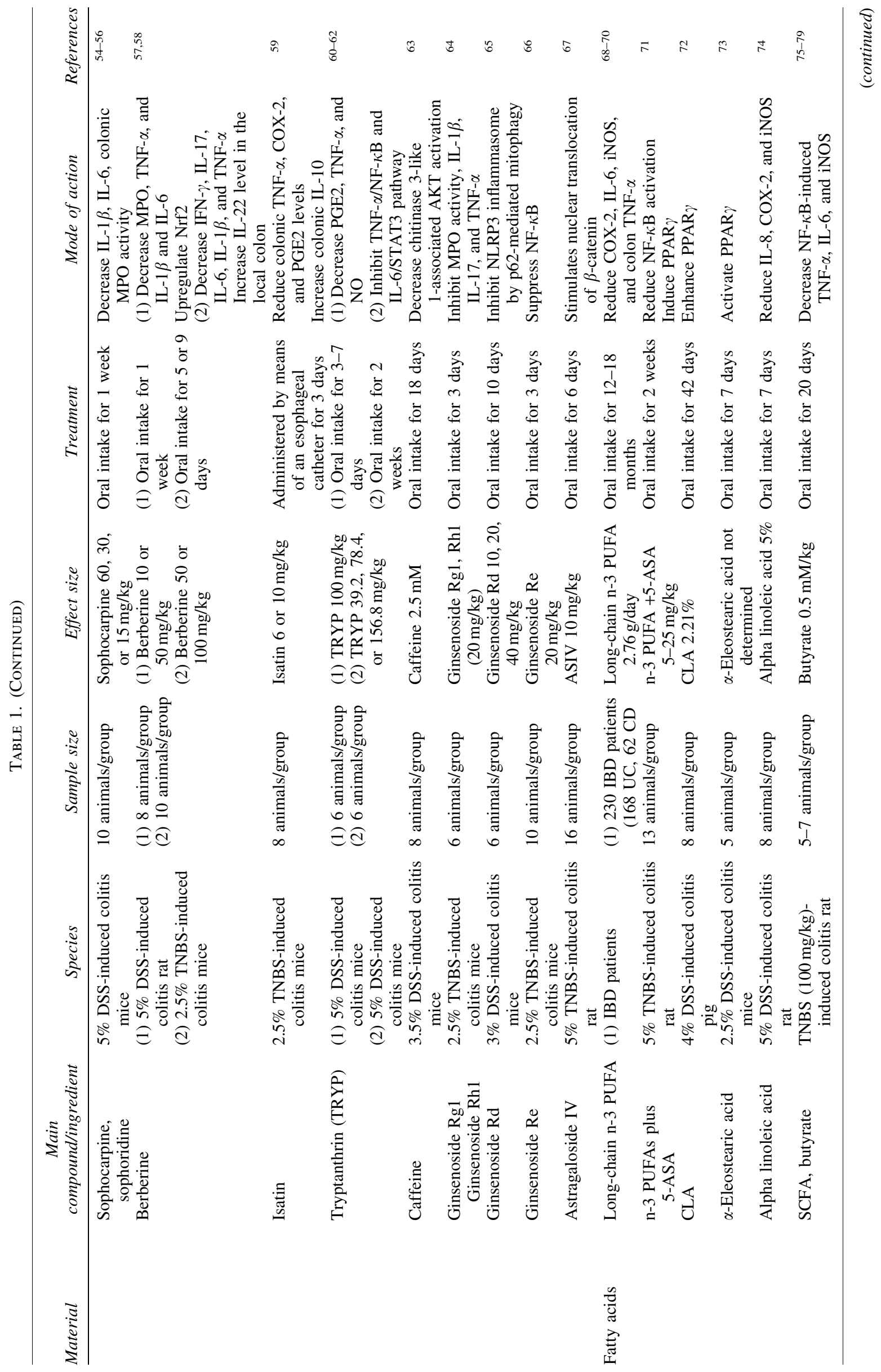




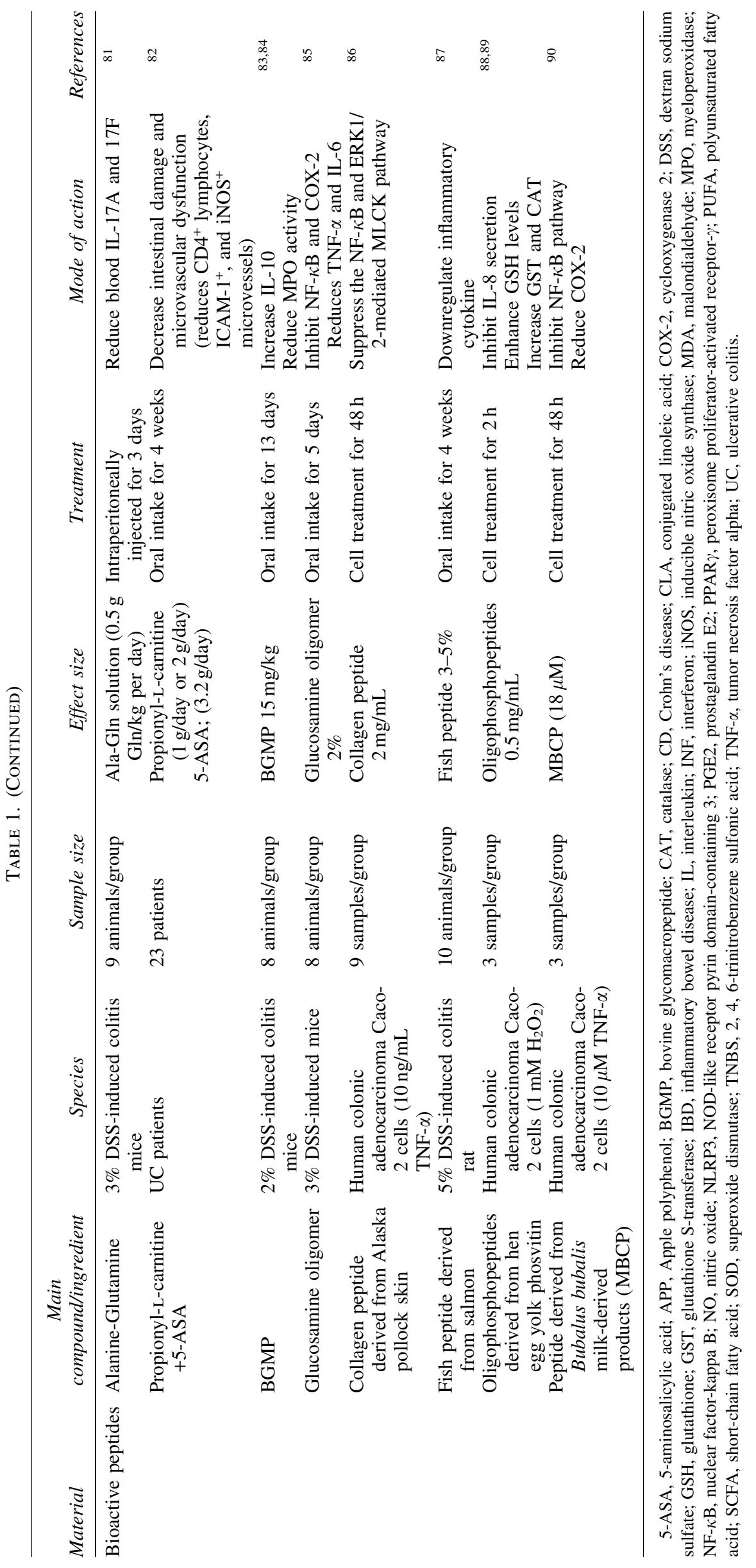




\section{Fatty acids}

Polyunsaturated fatty acids (PUFAs) reportedly modulate the immune response and contribute to ameliorating the symptoms of inflammatory diseases. The therapeutic effect of PUFAs on IBD has long been demonstrated, and the results suggest that dietary PUFA intake is to be a good strategy for preventing or improving IBD. ${ }^{68} \mathrm{~A}$ recent study has reported that a high dietary long-chain n-3 PUFAs intake reduces the risk of UC. ${ }^{69} \mathrm{~N}-3$ PUFA was shown to protect against intestinal inflammation in a rodent TNBS-induced colitis models. In addition, a recent study has demonstrated that n-3 PUFA significantly reduced COX-2, IL-6, colon iNOS, and colon $\mathrm{TNF}-\alpha$ production compared with the control diet, indicating that dietary n-3 PUFA can ameliorate colitis development by downregulating inflammatory markers. ${ }^{70}$ A previous study demonstrated that dual therapy of n-3 PUFAs plus 5aminosalicylic acid (5-ASA) is more effective than only 5ASA at reducing NF- $\kappa \mathrm{B}$ activation and inducing peroxisome proliferator-activated receptor- $\gamma(\operatorname{PPAR} \gamma)$ in a colitis model. ${ }^{71}$

A previous study concluded that conjugated linoleic acid (CLA) supplementation enhanced PPAR $\gamma$ expression in the colon and contributed to retarding the onset of IBD. CLA was tested in a clinical study of patients with CD. ${ }^{72}$ Dietary $\alpha$-eleostearic acid, which has been found in bitter gourd seed oils, improved the symptoms of IBD model mice by activating PPAR $\gamma \cdot{ }^{73}$ Alpha linoleic acid supplementation was shown to effectively inhibit IL- $1 \beta$-induced inflammation by downregulating mRNA levels of proinflammatory genes such as IL-8, COX-2, and iNOS. ${ }^{74}$

The number of bacteria-producing short-chain fatty acids (SCFA) was decreased in the mucous membranes and feces of patients with IBD. SCFAs, which mainly include acetate (C2), propionate (C3), and butyrate (C4), are necessary metabolites in the regulation of intestine homeostasis. Some studies have demonstrated that the levels of SCFA are significantly decreased in patients with IBD. SCFA plays a key role in the IECs and contributes to strengthening the intestinal barrier. ${ }^{75}$ Butyrate possesses beneficial effects for IECs and immune cells by increasing the levels of anti-inflammatory mediators (e.g., IL-10) but decreasing the expression of NF- $\kappa \mathrm{B}$-induced TNF- $\alpha$, IL- 6 , and iNOS. Butyrate also strengthens the epithelial barrier function by inducing tight junction components and contributes to protein reassembly by activating transcription factors such as STAT3 and SP1. ${ }^{76-79}$

\section{Bioactive peptides}

Dietary peptides derived from food possess bioactive properties that are healthful for preventing disease. ${ }^{80}$ Some bioactive peptides show potential for use as alternative IBD treatments. Post-treatment with alanineglutamine inhibited the expression of Th17-associated cytokines, decreased the peritoneal cavity of macrophage infiltration, and reduced the secretion of proinflammatory cytokine in DSS-induced colitis. ${ }^{81}$

The oral and intrarectal administration of propionyl-Lcarnitine, an ester of L-carnitine necessary for the mito- chondrial transport of fatty acids, significantly decreased intestinal impairment and microvascular dysfunction in rat TNBS-induced colitis. ${ }^{82}$ Bovine glycomacropeptide (BGMP), a 64-amino-acid peptide derived from the digestion of milk k-casein, showed immunomodulatory, antimicrobial, and possibly prebiotic activities. BGMP significantly reduced mucosal impairment in the colon and contributed to increasing IL-10 expression in lymphocyte-driven colitis. ${ }^{83}$ In addition, the oral administration of BGMP showed antiinflammatory effects in an experimental colitis model. ${ }^{84}$

Orally administered glucosamine oligomer exhibited anti-inflammatory effects by suppressing the activation of both NF- $\kappa \mathrm{B}$ and COX-2 in a DSS-induced colitis model. ${ }^{85}$ Collagen peptide derived from Alaska pollock skin ameliorated TNF- $\alpha$-induced barrier dysfunction in Caco- 2 cells by suppressing the NF- $\kappa \mathrm{B}$ and ERK1/2-mediated MLCK pathways. ${ }^{86}$ Fish peptide derived from salmon by enzymatic hydrolysis showed fewer signs of histopathological inflammation and downregulation of inflammatory cytokines in a DSS-induced colitis. ${ }^{87}$ Oligophosphopeptides derived from hen egg yolk inhibited the secretion of IL-8 in $\mathrm{H}_{2} \mathrm{O}_{2}$-induced Caco- 2 cells but enhanced the levels of GSH and increased catalase, glutathione S-transferase, and $\gamma$ glutamyl-cysteine synthetase activities. ${ }^{88,89}$ A peptide derived from Bubalus bubalis milk-derived products strengthened the organization of adherent epithelial junctions, inhibited the NF- $\kappa \mathrm{B}$ pathway, and decreased intestinal permeability. ${ }^{90}$

\section{CONCLUSIONS}

Although candidate disease-related targets of IBD are being found, the key factors have not been identified. Therefore, many kinds of research regarding the complex interaction among these disease-related targets should be continuously performed.

Long-term treatment of current drugs for curing IBD can cause side effects such as bone alterations or osteoporosis in the case of using glucocorticoids, and nutritional therapy may be an alternative intervention to improve IBD symptoms. In addition, the fact that IBD is predominant in westernized countries and that the number of patients is increasing in Asia, where recent western food is increasing, suggests that dietary modifications may be important for the treatment of IBD. As previously described, many functional foods and nutraceuticals are effective for inflammation and damaged barrier permeability, which are representative symptoms of IBD. In the future, human studies and clinical trials evaluating the best combinations of multiple functional foods and nutraceuticals, which possess antiinflammatory and/or immunomodulatory properties, will be important for improving IBD therapy.

\section{AUTHORS' CONTRIBUTIONS}

Writing and original draft preparation were by D.W.S. Writing, review, and editing were done by B.O.L. 


\section{AUTHOR DISCLOSURE STATEMENT}

No competing financial interests exist.

\section{FUNDING INFORMATION}

This study was supported by the Korea Institute of Planning and Evaluation for Technology in Food, Agriculture, Forestry, and Fisheries (IPET) through the High Value-added Food Technology Development Program, funded by the Ministry of Agriculture, Food and Rural Affairs (MAFRA; 117073-3).

\section{REFERENCES}

1. Pithadia AB, Jain S: Treatment of inflammatory bowel disease (IBD). Pharmacol Rep 2011;63:629-642.

2. Burisch J, Jess T, Martinato M, Lakatos PL, EpiCom E: The burden of inflammatory bowel disease in Europe. J Crohns Colitis 2013;7:322-337.

3. Bhandari S, Larson ME, Kumar N, Stein D: Association of inflammatory bowel disease (IBD) with depressive symptoms in the United States population and independent predictors of depressive symptoms in an IBD opulation: A NHANES Study. Gut Liver 2017;11:512-519.

4. Rizzello F, Spisni E, Giovanardi E, et al.: Implications of the westernized diet in the onset and progression of IBD. Nutrients 2019;11:E1033.

5. Mijan MA, Lim BO: Diets, functional foods, and nutraceuticals as alternative therapies for inflammatory bowel disease: Present status and future trends. World J Gastroenterol 2018;24:26732685.

6. Hur SJ, Kang SH, Jung HS, et al.: Review of natural products actions on cytokines in inflammatory bowel disease. Nutr Res 2012;32:801-816.

7. Durchschein F, Petritsch W, Hammer HF: Diet therapy for inflammatory bowel diseases: The established and the new. World J Gastroenterol 2016;22:2179-2194.

8. Paturi G, Mandimika T, Butts CA, et al.: Influence of dietary blueberry and broccoli on cecal microbiota activity and colon morphology in mdr1a(-/-) mice, a model of inflammatory bowel diseases. Nutrition 2012;28:324-330.

9. Hamer HM, Jonkers DM, Vanhoutvin SA, et al.: Effect of butyrate enemas on inflammation and antioxidant status in the colonic mucosa of patients with ulcerative colitis in remission. Clin Nutr 2010;29:738-744.

10. Le Leu RK, Young GP, Hu Y, Winter J, Conlon MA: Dietary red meat aggravates dextran sulfate sodium-induced colitis in mice whereas resistant starch attenuates inflammation. Dig Dis Sci 2013;58:3475-3482.

11. Lim HS, Kim SK, Hong SJ: Food elimination diet and nutritional deficiency in patients with inflammatory bowel disease. Clin Nutr Res 2018;7:48-55.

12. Farombi EO, Adedara IA, Ajayi BO, Ayepola OR, Egbeme EE: Kolaviron, a natural antioxidant and anti-inflammatory phytochemical prevents dextran sulphate sodium-induced colitis in rats. Basic Clin Pharmacol Toxicol 2013;113:49-55.

13. Ali AA, Abd Al Haleem EN, Khaleel SA, Sallam AS: Protective effect of cardamonin against acetic acid-induced ulcerative colitis in rats. Pharmacol Rep 2017;69:268-275.
14. Knight-Sepulveda K, Kais S, Santaolalla R, Abreu MT: Diet and inflammatory bowel disease. Gastroenterol Hepatol 2015;11: 511-520.

15. Ardizzone S, Bianchi Porro G: Biologic therapy for inflammatory bowel disease. Drugs 2005;65:2253-2286.

16. Horvath K, Varga C, Berko A, Posa A, Laszlo F, Whittle BJ: The involvement of heme oxygenase-1 activity in the therapeutic actions of 5-aminosalicylic acid in rat colitis. Eur J Pharmacol 2008;581:315-323.

17. Horiuchi T, Mitoma H, Harashima S, Tsukamoto H, Shimoda T: Transmembrane TNF-alpha: Structure, function and interaction with anti-TNF agents. Rheumatology (Oxford) 2010;49:12151228.

18. Garcia-Carbonell R, Wong J, Kim JY, et al.: Elevated A20 promotes TNF-induced and RIPK1-dependent intestinal epithelial cell death. Proc Natl Acad Sci U S A 2018;115:E9192E9200.

19. Chen L, Deng H, Cui H, et al:: Inflammatory responses and inflammation-associated diseases in organs. Oncotarget 2018;9: 7204-7218.

20. Lichtenstein GR: Comprehensive review: Antitumor necrosis factor agents in inflammatory bowel disease and factors implicated in treatment response. Therap Adv Gastroenterol 2013;6: 269-293.

21. Kumar A, Takada Y, Boriek AM, Aggarwal BB: Nuclear factorkappaB: Its role in health and disease. J Mol Med 2004;82:434448.

22. McDaniel DK, Eden K, Ringel VM, Allen IC: Emerging roles for noncanonical NF-kappaB signaling in the modulation of inflammatory bowel disease pathobiology. Inflamm Bowel Dis 2016;22:2265-2279.

23. Rahman SU, Li Y, Huang Y, et al.: Treatment of inflammatory bowel disease via green tea polyphenols: Possible application and protective approaches. Inflammopharmacol 2018;26:319330.

24. Yao X, Huang J, Zhong H, et al.: Targeting interleukin-6 in inflammatory autoimmune diseases and cancers. Pharmacol Ther 2014;141:125-139.

25. Shipman L: Rheumatoid arthritis: Tocilizumab and the risk of intestinal perforation. Nat Rev Rheumatol 2016;12:499.

26. Brand S, Beigel F, Olszak T, et al.: IL-22 is increased in active Crohn's disease and promotes proinflammatory gene expression and intestinal epithelial cell migration. Am J Physiol 2006;290: G827-G838.

27. Zenewicz LA, Flavell RA: Recent advances in IL-22 biology. Int Immunol 2011;23:159-163.

28. Mizoguchi A: Healing of intestinal inflammation by IL-22. Inflamm Bowel Dis 2012;18:1777-1784.

29. Andoh A, Shioya M, Nishida A, et al:: Expression of IL-24, an activator of the JAK1/STAT3/SOCS3 cascade, is enhanced in inflammatory bowel disease. J Immunol 2009;183:687-695.

30. Mizoguchi A, Yano A, Himuro H, Ezaki Y, Sadanaga T, Mizoguchi E: Clinical importance of IL-22 cascade in IBD. J Gastroenterol 2018;53:465-474.

31. Begue B, Verdier J, Rieux-Laucat F, et al.: Defective IL10 signaling defining a subgroup of patients with inflammatory bowel disease. Am J Gastroenterol 2011;106:1544-1555.

32. Ito K, Chung KF, Adcock IM: Update on glucocorticoid action and resistance. J Allergy Clin Immunol 2006;117:522-543. 
33. Renna S, Cottone M, Orlando A: Optimization of the treatment with immunosuppressants and biologics in inflammatory bowel disease. World J Gastroenterol 2014;20:9675-9690.

34. Crowe JS, Roberts KJ, Carlton TM, et al.: Preclinical development of a novel, orally-administered anti-tumour necrosis factor domain antibody for the treatment of inflammatory bowel disease. Sci Rep 2018;8:4941.

35. Nitzan O, Elias M, Peretz A, Saliba W: Role of antibiotics for treatment of inflammatory bowel disease. World J Gastroenterol 2016;22:1078-1087.

36. Moura FA, de Andrade KQ, Dos Santos JCF, Araujo ORP, Goulart MOF: Antioxidant therapy for treatment of inflammatory bowel disease: Does it work? Redox Biol 2015;6:617-639.

37. Roth S, Spalinger MR, Gottier C, et al.: Bilberry-derived anthocyanins modulate cytokine expression in the intestine of $\mathrm{Pa}-$ tipents with ulcerative colitis. PLoS One 2016;11:e0154817.

38. Park MY, Ji GE, Sung MK: Dietary kaempferol suppresses inflammation of dextran sulfate sodium-induced colitis in mice. Dig Dis Sci 2012;57:355-363.

39. Dodda D, Chhajed R, Mishra J: Protective effect of quercetin against acetic acid induced inflammatory bowel disease (IBD) like symptoms in rats: Possible morphological and biochemical alterations. Pharmacol Rep 2014;66:169-173.

40. Cibicek N, Roubalova L, Vrba J, et al.: Protective effect of isoquercitrin against acute dextran sulfate sodium-induced rat colitis depends on the severity of tissue damage. Pharmacol Rep 2016;68:1197-1204.

41. Marquez-Flores YK, Villegas I, Cardeno A, Rosillo MA, Alarconde-la-Lastra C: Apigenin supplementation protects the development of dextran sulfate sodium-induced murine experimental colitis by inhibiting canonical and non-canonical inflammasome signaling pathways. J Nutr Biochem 2016;30:143-152.

42. Xu L, Yang ZL, Li P, Zhou YQ: Modulating effect of Hesperidin on experimental murine colitis induced by dextran sulfate sodium. Phytomedicine 2009;16:989-995.

43. Kang GD, Kim DH: Poncirin and its metabolite ponciretin attenuate colitis in mice by inhibiting LPS binding on TLR4 of macrophages and correcting Th17/Treg imbalance. J Ethnopharmacol 2016;189:175-185.

44. Zhao J, Hong T, Dong M, Meng Y, Mu J: Protective effect of myricetin in dextran sulphate sodium-induced murine ulcerative colitis. Mol Med Rep 2013;7:565-570.

45. Al-Rejaie SS, Abuohashish HM, Al-Enazi MM, Al-Assaf AH, Parmar MY, Ahmed MM: Protective effect of naringenin on acetic acid-induced ulcerative colitis in rats. World J Gastroenterol 2013;19:5633-5644.

46. Azuma T, Shigeshiro M, Kodama M, Tanabe S, Suzuki T: Supplemental naringenin prevents intestinal barrier defects and inflammation in colitic mice. J Nutr 2013;143:827-834.

47. Kumar VS, Rajmane AR, Adil M, Kandhare AD, Ghosh P, Bodhankar SL: Naringin ameliorates acetic acid induced colitis through modulation of endogenous oxido-nitrosative balance and DNA damage in rats. J Biomed Res 2014;28:132-145.

48. Skyberg JA, Robison A, Golden S, et al:: Apple polyphenols require $\mathrm{T}$ cells to ameliorate dextran sulfate sodium-induced colitis and dampen proinflammatory cytokine expression. J Leukoc Biol 2011;90:1043-1054.

49. Martin AR, Villegas I, Sanchez-Hidalgo M, de la Lastra CA: The effects of resveratrol, a phytoalexin derived from red wines, on chronic inflammation induced in an experimentally induced colitis model. Br J Pharmacol 2006;147:873-885.

50. Youn J, Lee JS, Na HK, Kundu JK, Surh YJ: Resveratrol and piceatannol inhibit iNOS expression and NF-kappaB activation in dextran sulfate sodium-induced mouse colitis. Nutr Cancer 2009;61:847-854.

51. Samsami-Kor M, Daryani NE, Asl PR, Hekmatdoost A: Antiinflammatory effects of resveratrol in patients with ulcerative colitis: A randomized, double-blind, placebo-controlled pilot study. Arch Med Res 2015;46:280-285.

52. Liu L, Liu YL, Liu GX, et al.: Curcumin ameliorates dextran sulfate sodium-induced experimental colitis by blocking STAT3 signaling pathway. Int Immunopharmacol 2013;17:314-320.

53. Gong Z, Zhao S, Zhou J, et al.: Curcumin alleviates DSS-induced colitis via inhibiting NLRP3 inflammsome activation and IL1beta production. Mol Immunol 2018;104:11-19.

54. Bunsupa S, Yamazaki M, Saito K: Quinolizidine alkaloid biosynthesis: Recent advances and future prospects. Front Plant Sci 2012;3:239.

55. Zhang YB, Yang L, Luo D, et al.: Sophalines E-I, five quinolizidine-based alkaloids with antiviral activities against the hepatitis B virus from the seeds of sophora alopecuroides. Org Lett 2018;20:5942-5946.

56. Wang XJ, Deng HZ, Jiang B, Yao H: The natural plant product sophocarpine ameliorates dextran sodium sulfate-induced colitis in mice by regulating cytokine balance. Int $J$ Colorectal Dis 2012;27:575-581.

57. Jing W, Safarpour Y, Zhang T, et al.: Berberine upregulates p-glycoprotein in human caco-2 cells and in an experimental model of colitis in the rat via activation of Nrf2-dependent mechanisms. J Pharmacol Exp Ther 2018;366:332-340.

58. Li C, Xi Y, Li S, et al:: Berberine ameliorates TNBS induced colitis by inhibiting inflammatory responses and Th1/Th17 differentiation. Mol Immunol 2015;67:444-454.

59. Socca EA, Luiz-Ferreira A, de Faria FM, et al.: Inhibition of tumor necrosis factor-alpha and cyclooxigenase-2 by Isatin: A molecular mechanism of protection against TNBS-induced colitis in rats. Chem Biol Interact 2014;209:48-55.

60. Lee S, Kim DC, Baek HY, Lee KD, Kim YC, Oh H: Antineuroinflammatory effects of tryptanthrin from Polygonum tinctorium Lour. in lipopolysaccharide-stimulated BV2 microglial cells. Arch PHarmacal Res 2018;41:419-430.

61. Micallef MJ, Iwaki $\mathrm{K}$, Ishihara $\mathrm{T}$, et al.: The natural plant product tryptanthrin ameliorates dextran sodium sulfate-induced colitis in mice. Int Immunopharmacol 2002;2:565-578.

62. Wang Z, Wu X, Wang CL, et al.: Tryptanthrin protects mice against dextran sulfate sodium-induced colitis through inhibition of TNF-alpha/NF-kappaB and IL-6/STAT3 pathways. Molecules 2018;23:E1062.

63. Lee IA, Low D, Kamba A, Llado V, Mizoguchi E: Oral caffeine administration ameliorates acute colitis by suppressing chitinase 3-like 1 expression in intestinal epithelial cells. $J$ Gastroenterol 2014;49:1206-1216.

64. Lee SY, Jeong JJ, Eun SH, Kim DH: Anti-inflammatory effects of ginsenoside $\mathrm{Rg} 1$ and its metabolites ginsenoside $\mathrm{Rh} 1$ and 20(S)-protopanaxatriol in mice with TNBS-induced colitis. Eur J Pharmacol 2015;762:333-343.

65. Liu C, Wang J, Yang Y, et al.: Ginsenoside Rd ameliorates colitis by inducing p62-driven mitophagy-mediated NLRP3 
inflammasome inactivation in mice. Biochem Pharmacol 2018; 155:366-379.

66. Lee IA, Hyam SR, Jang SE, Han MJ, Kim DH: Ginsenoside Re ameliorates inflammation by inhibiting the binding of lipopolysaccharide to TLR4 on macrophages. J Agric Food Chem 2012; 60:9595-9602.

67. Jiang XG, Sun K, Liu YY, et al.: Astragaloside IV ameliorates 2,4,6-trinitrobenzene sulfonic acid (TNBS)-induced colitis implicating regulation of energy metabolism. Sci Rep 2017;7: 41832 .

68. Uchiyama K, Nakamura M, Odahara S, et al.: N-3 polyunsaturated fatty acid diet therapy for patients with inflammatory bowel disease. Inflamm Bowel Dis 2010;16:1696-1707.

69. Mozaffari H, Daneshzad E, Larijani B, Bellissimo N, Azadbakht L: Dietary intake of fish, n-3 polyunsaturated fatty acids, and risk of inflammatory bowel disease: A systematic review and metaanalysis of observational studies. Eur J Nutr 2020;59:1-17.

70. Charpentier C, Chan R, Salameh E, et al.: Dietary n-3 PUFA may attenuate experimental colitis. Med Inflamm 2018;2018:8430614.

71. Mbodji K, Charpentier C, Guerin C, et al.: Adjunct therapy of n-3 fatty acids to 5-ASA ameliorates inflammatory score and decreases NF-kappaB in rats with TNBS-induced colitis. $J$ Nutr Biochem 2013;24:700-705.

72. Bassaganya-Riera J, Hontecillas R: CLA and n-3 PUFA differentially modulate clinical activity and colonic PPAR-responsive gene expression in a pig model of experimental IBD. Clin Nutr 2006;25:454-465.

73. Lewis SN, Brannan L, Guri AJ, et al.: Dietary alpha-eleostearic acid ameliorates experimental inflammatory bowel disease in mice by activating peroxisome proliferator-activated receptorgamma. PLoS One 2011;6:e24031.

74. Reifen R, Karlinsky A, Stark AH, Berkovich Z, Nyska A: AlphaLinolenic acid (ALA) is an anti-inflammatory agent in inflammatory bowel disease. J Nutr Biochem 2015;26:1632-1640.

75. Goncalves P, Araujo JR, Di Santo JP: A cross-talk between microbiota-derived short-chain fatty acids and the host mucosal immune system regulates intestinal homeostasis and inflammatory bowel disease. Inflamm Bowel Dis 2018;24:558-572.

76. Cushing K, Alvarado DM, Ciorba MA: Butyrate and mucosal inflammation: New scientific evidence supports clinical observation. Clin Transl Gastroenterol 2015;6:e108.

77. Zhang M, Zhou Q, Dorfman RG, et al.: Butyrate inhibits interleukin-17 and generates Tregs to ameliorate colorectal colitis in rats. BMC Gastroenterol 2016;16:84.

78. Segain JP, Raingeard de la Bletiere D, Bourreille A, et al.: Butyrate inhibits inflammatory responses through NFkappaB inhibition: Implications for Crohn's disease. Gut 2000;47:397-403.
79. Parada Venegas D, De la Fuente MK, Landskron G, et al:: Short chain fatty acids (SCFAs)-mediated gut epithelial and immune regulation and its relevance for inflammatory bowel diseases. Front Immunol 2019;10:277.

80. Chakrabarti S, Jahandideh F, Wu J: Food-derived bioactive peptides on inflammation and oxidative stress. BioMed Res Int 2014;2014:608979.

81. Hou YC, Liu JJ, Pai MH, Tsou SS, Yeh SL: Alanyl-glutamine administration suppresses Th17 and reduces inflammatory reaction in dextran sulfate sodium-induced acute colitis. Int Immunopharmacol 2013;17:1-8.

82. Scioli MG, Stasi MA, Passeri D, et al.: Propionyl-L-carnitine is efficacious in ulcerative colitis through its action on the immune function and microvasculature. Clin Transl Gastroenterol 2014; 5:e55.

83. Ortega-Gonzalez M, Capitan-Canadas F, Requena P, et al.: Validation of bovine glycomacropeptide as an intestinal antiinflammatory nutraceutical in the lymphocyte-transfer model of colitis. Br J Nutr 2014;111:1202-1212.

84. Requena P, Daddaoua A, Martinez-Plata E, et al.: Bovine glycomacropeptide ameliorates experimental rat ileitis by mechanisms involving downregulation of interleukin 17. $\mathrm{Br} J$ Pharmacol 2008;154:825-832.

85. Azuma K, Osaki T, Kurozumi S, et al: Anti-inflammatory effects of orally administered glucosamine oligomer in an experimental model of inflammatory bowel disease. Carbohydr Polym 2015;115:448-456.

86. Chen Q, Chen O, Martins IM, et al.: Collagen peptides ameliorate intestinal epithelial barrier dysfunction in immunostimulatory Caco- 2 cell monolayers via enhancing tight junctions. Food Funct 2017;8:1144-1151.

87. Grimstad T, Bjorndal B, Cacabelos D, et al.: A salmon peptide diet alleviates experimental colitis as compared with fish oil. J Nutr Sci 2013;2:e2.

88. Katayama S, Xu X, Fan MZ, Mine Y: Antioxidative stress activity of oligophosphopeptides derived from hen egg yolk phosvitin in Caco-2 cells. J Agric Food Chem 2006;54:773778 .

89. Katayama S, Ishikawa S, Fan MZ, Mine Y: Oligophosphopeptides derived from egg yolk phosvitin up-regulate gamma-glutamylcysteine synthetase and antioxidant enzymes against oxidative stress in caco-2 cells. J Agric Food Chem 2007; 55:2829-2835.

90. Tenore GC, Pagano E, Lama S, et al: Intestinal antiinflammatory effect of a peptide derived from gastrointestinal digestion of buffalo (Bubalus bubalis) mozzarella cheese. $\mathrm{Nu}$ trients 2019;11:E610. 